\title{
Mental Health Status \& Stress: A Comparative Study among Yoga- doer and Yoga- non doer Older Adults
}

\author{
Dr. Krishna J. Vaghela 1
}

\begin{abstract}
ABATRACT
The present study has been designed to investigate the difference of mental health status and stress among yoga- doer and yoga - non doer older adults. The research was conducted over a sample of 120 older adults both yoga - doer and yoga - non doer as well as male and female.[30 yoga - doer and 30 yoga - non doer male older adults and 30 yoga - doer and 30 - yoga non doer female older adults.] All the participants were administered the mental health inventory and stress inventory. Data was analyzed using $\mathrm{t}$ test. The results reported that there exists a significant difference in mental health status among yoga- doer and yoga - non doer older adults both male and female. Yoga - doer older adults had better mental as compare to yoga - non doer older adults. Significant difference is also observed between yoga- doer and yoga - non doer older adults as regarding to their level of stress scores. Yoga also helpful in reduce stress level.
\end{abstract}

Keywords: Yoga, Mental health, Stress, Yoga-doer, Yoga-non doer, Older adults.

\section{INTRODUCTION:}

As the number of older adults is growing, so will mental health problems. Mental health disorders including stress, anxiety, depression, adversely affect physical health and ability to function, especial in older adults. Physical and mental health affect each other for example, older adults with medical problems such as heart disease have higher rates of stress, depression than those who are medically well. Stress in older adults is a very treatable disorder. Stress is a major factor affecting the mental health of older adults. Conversely untreated stress in an older person with heart disease negatively affects the outcome of the disease. Older adults those aged 60 or above are at risk of developing mental disorders, neurological disorders or substance use problems as well as physical illness or disability.

Mental and physical health problems can also interact in older people making their overall assessment and management more difficult. In general older people feel less happy with their life than younger people and this is of special concern in new number states where there are larger differences in life stratification and happiness between age groups. There are no agreed definitions of when 'old age' begins and the common understanding of belonging to older age groups has different connotations and meanings across cultures, societies and peoples.

${ }^{1}$ Asso. Prof. \& Head, Dept. of Psychology, Yogiji Maharaj Mahavidyalaya, Dhari

(C) 2015, K Vaghela; licensee IJIP. This is an Open Access Research distributed under the terms of the Creative Commons Attribution License (http://creativecommons.org/licenses/by/2.0), which permits unrestricted use, distribution, and reproduction in any Medium, provided the original work is properly cited. 


\section{Mental Health Status \& Stress: A Comparative Study among Yoga- doer and \\ Yoga- non doer Older Adults}

Stress :- Stress is a pressure or demand on the system where available resources are not adequate to cope with. Stress can disturb any one's physical, mental, emotional and behavioral balance. Stress can damage different parts of human body muscles and tissues to organs and blood vessels, blood pressure, body temperature. It can also interfere with the body metabolism, digestion, sleep, fertility, appetite, sexuality etc.

Yoga :- 'Yoga is ancient that is defined as the union of the soul with God'. 'Yoga is a path of personal, spiritual development that utilizes meditation to bring enlightenment, self-realization and ultimately, the attainment of god and bliss.'

Yoga is not only sate for older petitioners, but also effective in keeping the mind and body in good health. Yoga is an ancient Indian science which helps to improve physical, mental, social and spiritual health. Yoga has been found useful for mental disorders like - depression, stress. Yoga helps to improve the mental health of both the yoga and seniors by reducing stress. Many older people also experience functional limitations due to health related problems. Estimates suggest that up to $40 \%$ of the hearing impaired population many experience mental health difficulties at some time in their lives. Other functional impairments also lead to depression around $20 \%$ in some studies. Older women are more likely than mint to experience negative affects on social activities caused by their physical or mental health problems. Researchers confirm that older adults with evidence of mental disorder are less likely then younger and middle aged adults to receive mental health services and that, when they do, they are less likely to receive care from a mental health specialist (Karel, Gatz \& Smyer 2012). A study of 149 persons with non-insulin dependent diabetes found that 104 had lowered blood sugar and needed less oral anti diabetes medication after regularly practicing yoga. Researchers at Bhabha Atomic Research centre, medical division in Mumbai India, evaluated the overall benefits of yoga on risk factors for heart disease (Damodaran, Mulathi, Patil, Shah, Suryavansihi, Marathe 2002). A group of 20 patients age 35 to 55 above years of age, all of whom had mild to moderate high blood pressure began a daily one hour yoga program prior to the implementation of their yoga program and following three months of yoga, biochemical and psychological parameters were studies. The overall results were quite impressive. After the three months of yoga practice, the patients experienced a decrease in blood pressure, as well as a decrease in blood sugar, cholesterol and triglycerides. Feedback also indicated that the patients were calmer.

A study of the university, college of medical science in New Delhi, evaluated 30 to 60 years old patients with type 11 diabetes (Jain, Uppal, Bhatnagar, Talukder 1993). A 40 minute per day regimen to yoga was followed for a period of 40 day. The results showed a significant decrease in fasting blood sugar levels. A British study of 71 healthy volunteers aged 21 to 76 found that a 30 minute program of yogic stretching and breathing exercises was simple to learn and resulted in a "markedly invigorating" effect on perceptions of both mental and physical energy and improved mood. Some expert suggest that the regular practice of breathing through one nostril 


\section{Mental Health Status \& Stress: A Comparative Study among Yoga- doer and \\ Yoga- non doer Older Adults}

may help improve communication between the right and left side of brain. Yoga can number of benefits for people over 50, from healthy bones, to flexibility, to stress relief.

The harbor - UCLA medical centre conducted a study to assess what effects, if any, yoga has on stress levels. (Gaur 2001) During the study, all participants expressed that their moods and anxiety levels were improved as a results of their yoga sessions. Researchers estimate that almost two third of older adults with a mental disorders. Therefore the aim of the present research was to find out the difference of mental health status and stress among yoga doer and yoga non-doer older adults.

\section{AIMS AND OBJECTIVES}

The main purpose of the present research was to find out the difference of mental health status and stress yoga doer and yoga non doer older adults. Some of the major objectives were framed to study the problem.

1. To examine the mental health status in yoga - doer and yoga - non doer male older adults.

2. To examine the mental health status in yoga - doer and yoga- non doer female older adults.

3. To examine the stress in yoga - doer and yoga - non doer male older adults.

4. To examine the stress in yoga - doer and yoga - non doer female older adults.

\section{HYPOTHESIS}

Keeping view the objectives of the present study following null hypotheses framed for the present research.

1. There is no significant difference in the mean scores of mental health status among yoga doer and yoga - non doer male older adults.

2. There is no significant difference in the mean scores of mental health status among yoga doer and yoga - non doer female older adults.

3. - There is no significant difference in the mean scores of stress among yoga - doer and yoga - non doer male older adults.

4. - There is no significant difference in the mean scores of stress among yoga - doer and yoga - non doer female older adults.

\section{RESEARCH METHODOLOGY}

In the present study achieve the above cited objectives, survey method was conducted by the researcher to collect relevant data regarding the present study. 


\section{Mental Health Status \& Stress: A Comparative Study among Yoga- doer and \\ Yoga- non doer Older Adults}

\section{Participants}

The sample was consisted of 120 older adults including yoga - doer and yoga - non doer both male and female older adults ranging in age from 65 to 80 years. The sample was selected through purposive and convenient sampling technique from Junagadh city. The sample was consists of only married older adults. A total of 120 older adults of which 60 were male older adults (30 yoga - doer and 30 non yoga - non doer) and 60 were female older adults (30 yoga - doer and 30 non yoga - non doer). All the participants were matched on the variables of age, area, marital status etc. The data has been collected with the help of questionnaires.

\section{Instruments}

The following standardized tools were used for the present investigation. Personal information schedule developed by investigator was used to collected some necessary information like, age gender, marital status etc. To measure mental health status, The Mental Hygiene Inventory developed by Bhatt \& Gida was used. Reliability of the test is 0.87 . And to measure stress, Stress Measurement Inventory by Bhatt, J. was used. The reliability and validity of the test is $0.91 \&$ 0.87

\section{Procedure}

The present research conduct on 120 older adults. After establishing report with the participants the questionnaires were administrated with the necessary instruction and the data was collected. All the participants were assured that their responses would be kept confidential. Scoring was done accorded the instructions given in the manual. The data was coded numerically to protected the participants privacy. Finally obtained results were statistically analyzed and discussed accordingly.

\section{Statistical Analysis}

The researcher put the data edited and coded together in a carefully designed table for statistical analysis t test was applied to see the significance of the difference between yoga - doer and yoga - non doer older adults on the basis of their mental health and stress scores.

\section{RESULTS \& DISCUSSION}

Applying t-test on the samples data analysis and interpretations. The main purpose of the present research was to find out the difference of mental health status and stress among yoga-doer and yoga-non doer older adults. The research findings are based on the responses of 120 older adults both male \& female as well as yoga-doer and yoga non-doer. Hol "There is no significant difference in the mean score of mental health status of yoga-doer and yoga non-doer male older adults," to assess this hypothesis t-test was used. Table -1 gives the descriptive statistics for 


\section{Mental Health Status \& Stress: A Comparative Study among Yoga- doer and \\ Yoga- non doer Older Adults}

each of two groups on mental health status (as defined by the yoga-doer and yoga non-doer). This t-test revealed statistically significant difference between the mean number of two groups, were group 1 has $(M=74.92)$ and group 2 has $(M=68.07)$ from table -1 reveal that there is significant difference in the mean scores of mental health status of comparative groups as the tvalue ( 2.63 ) is found to be significant at 0.01 level. Hence the null hypothesis is rejected and, it clearly indicates that male yoga-doer older adults had better mental health as compare to yoga non doer male older adults.

Ho2 "There is no significant difference in the mean scores of mental health status among yogadoer and yoga non-doer female older adults. Results from table -2 the mean value of mental health status in the female yoga-doer older adults (M 73.46) is greater than that of yoga-nondoer female older adults $(M=66.89)$. The result of t-test applied between the mean scores of mental health status of comparative groups indicates that they differ statistically significant as the t-value of t-test is (2.59). Thus, the null hypothesis also rejected and it is also found that mental health in female yoga-doer older adults had better than comparative group.

Table -1 : showing results of $t$-value of mental health status of yoga-doer and yoga non-doer male older adults

\begin{tabular}{|l|l|l|l|l|l|}
\hline Older Adults & No. & Mean & SD & t-value & Sig. \\
\hline Yoga-doer & 30 & 74.92 & 10.8 & 2.63 & 0.01 \\
\hline Yoga-non-doer & 30 & 68.07 & 9.3 & & \\
\hline
\end{tabular}

Table -2 : showing results of t-value of mental health status of yoga-doer and yoga non-doer female older adults

\begin{tabular}{|l|l|l|l|l|l|}
\hline Older Adults & No. & Mean & SD & t-value & Sig. \\
\hline Yoga-doer & 30 & 73.46 & 10.7 & 2.59 & 0.01 \\
\hline Yoga-non-doer & 30 & 66.89 & 8.9 & & \\
\hline
\end{tabular}

The research findings are based on the responses of 120 older adults both male and female on stress measurement inventory. (Both yoga - doer and yoga-non-doer) Ho3 "There is no significant difference in the mean score of stress of yoga - doer and yoga -non doer male older adults," to assess this hypothesis t-test was used. Results from table -3 t-test revealed a statistically significant difference in the mean scores of stress of two comparative groups, where group -1 has $(M=69.07)$ and group -2 has $(M=76.89)$. Thus $t$-test revealed statistically significant difference in the stress of yoga-doer and yoga-non-doer male older adults as the tvalue ( 2.23 ) is found to be significant at 0.05 level. Hence the null hypothesis is rejected and it clearly indicates that yoga-non doer male participants' responses showed high stress as compare the yoga-doer male older adults. 


\section{Mental Health Status \& Stress: A Comparative Study among Yoga- doer and \\ Yoga- non doer Older Adults}

Ho4 "There is no significant difference in the mean score of stress of yoga-doer and yoga non doer female older adults." To assess this hypothesis t-test was used, results showed in table -4 that there is significant difference between to comparative groups in respect to their stress scores. The obtained t-value is 2.01 which is significant at 0.05 level. Thus the null hypothesis is also rejected and it clearly indicate that from table -4 the mean value of stress in the female yoganon doer older adults $(\mathrm{M}=77.36)$ is greater as compare the yoga-doer female older adults $(\mathrm{M}=$ 70.11). It is concluded that yogic practices also reduce stress in yoga doer participants. In conclusion the present study indicating that yoga interventions can improve the mental health of older adults both: male and female, as well as yoga also helpful in management of stress, reduce of stress level.

Table -3 : showing results of t-value of stress level of yoga-doer and yoga non-doer male older adults

\begin{tabular}{|l|l|l|l|l|l|}
\hline Older Adults & No. & Mean & SD & t-value & Sig. \\
\hline Yoga- doer & 30 & 69.07 & 13.11 & \multirow{2}{*}{2.23} & \multirow{2}{*}{0.05} \\
\cline { 1 - 5 } Yoga-non doer & 30 & 76.89 & 14.02 & & \\
\hline
\end{tabular}

Table -4 : showing results of $t$-value of stress level of yoga-doer and yoga non-doer female older adults

\begin{tabular}{|l|l|l|l|l|l|}
\hline Older Adults & No. & Mean & SD & t-value & Sig. \\
\hline Yoga-doer & 30 & 70.11 & 13.20 & \multirow{2}{*}{2.01} & \multirow{2}{*}{0.05} \\
\cline { 1 - 5 } Yoga-non doer & 30 & 77.36 & 14.86 & & \\
\hline
\end{tabular}

\section{CONCLUSION}

The main purpose of the present research was to find out the difference of mental health status and stress yoga doer and yoga non doer older adults both male and female. After analysis and interpretation the following conclusion was drawn.

The mental health of male yoga - doer participants is significantly better than that of male yoga - non doer participants.

The mental health of female yoga - doer participants is also significantly better as compare the female yoga - non doer participants.

The male yoga - doer had low level of stress than male yoga - non doer participants.

The female yoga - doer had low level of stress as compare the female yoga - non doer participants. 


\section{REFERENCES}

1. Bhatt, D.J. \& Gida, G. (1992) The mental hygiene inventory MHI, construction and standardization, department of psychology, Saurashtra University, Rajkot.

2. Friedman, H.J. (2002) Health Psychology, prentice hall, New Jersory.

3. Garret, H. E. (1971) Statistics in psychology and education, veils Feiffer and simons private ltd. $6^{\text {th }}$ Indian addition Mumbai.

4. Kalpan, H.B. (1996) Psychological stress, New York : Academic press.

5. Mangal, S. K. (1992) Statistics in Psychology and Education, Tata, McGraw Hill Pub., Company Ltd. New Delhi.

6. Salaman, A (2000) Book review on mental health, Jefferson medical, college, Philadephia.

7. Shukla, K.C. (2005) Encyclopedic dictionary of psychology, vol. 1-3, pub. Ajay Verma for commonwealth publishers, Darya Gani, New Delhi. 